\title{
A randomized trial of multimedia-facilitated informed consent for cataract surgery
}

This article was published in the following Dove Press journal:

Clinical Ophthalmology

\section{Thomas A Vo \\ Philip Ngai \\ Jeremiah $\mathrm{P}$ Tao}

Department of Ophthalmology, Gavin Herbert Eye Institute, University of California Irvine School of Medicine, Irvine, CA, USA
Correspondence: Philip Ngai Gavin Herbert Eye Institute, University of California, Irvine, 850 Health Sciences Road, Irvine, CA 92697-4375, USA

Tel + I 9498242020

Email pngai@uci.edu
Objective: The aim of this study was to evaluate the value and role of patient's education videos in the informed consent process for patients undergoing preoperative assessment of cataracts. Design: The study is a single-center prospective randomized controlled trial.

Subjects, participants, and/or controls: Participants enrolled in this study were specifically those undergoing first-time phacoemulsification cataract surgery with the placement of a monofocal lens implant.

Participants and methods: Subjects were randomized to either face-to-face surgeoninformed consent with a preceding education video or face-to-face surgeon-informed consent alone.

Main outcome measures: The main outcome measures assessed were time to complete the informed consent process, patient's satisfaction, and patient's comprehension.

Results: The video and control groups were similar in satisfaction $(4.67 \pm 0.104$ video vs. $4.53 \pm 0.133$ control; $P=0.43)$ and comprehension $(79.4 \% \pm 2.82 \%$ video vs. $79.3 \% \pm 3.39 \%$ control; $P=0.99)$. Counseling time was statistically significantly different $(117.5 \pm 10.9$ seconds video versus 241.6 \pm 13.0 seconds control; $P<0.0001)$.

Conclusion: Use of a patient's education video for cataract surgery was associated with reduced physician counseling time yet similar comprehension and patient-reported satisfaction when compared with traditional counseling methods.

Keywords: video, cataract surgery, informed consent, quality improvement, patient's education

\section{Introduction}

Cataracts are the most common cause of blindness worldwide, with more than half of all Americans developing cataracts by the age of 80 years. ${ }^{1,2}$ Associated with aging, the clouding lens can severely degrade the quality of life. Activities of daily living such as reading and driving can become challenging or even impossible; however, the overwhelming majority of cataract removal surgeries are efficient and relatively low risk. As the elderly population continues to grow; the demand for cataract surgery will also rise. Unsurprisingly, cataract surgery is the most commonly performed surgery in the USA. ${ }^{3}$ Delays in the preoperative assessment, because of longer wait times in clinic or appointment delays, prolong patient's anxiety. Previous studies have demonstrated that the surgical informed consent process was effective in reducing patient's anxiety, therefore highlighting the need to optimize the preoperative workup. ${ }^{4}$

The use of digital media for educating patients about surgery is becoming more widely used. ${ }^{5-10}$ The ability to deliver the necessary information about the risks and benefits of surgery in a consistent but timely manner offers an appealing advantage. Patients are also able to view and review the videos at their leisure, which allows 
them time to formulate questions and concerns prior to their preoperative appointment. Video sharing websites, such as YouTube (Google LLC, San Mateo, CA, USA), have become increasingly popular resources for learning more about medical procedures. Previous studies have found that videos can increase the retention of knowledge by $\sim 25 \%{ }^{11}$ and help reduce anxiety, increased preparedness, and improve overall satisfaction. ${ }^{6-8}$

Here, we performed a single-center randomized trial evaluating whether the use of a cataract surgery education video shown before receiving face-to-face counseling from a surgeon compared with receiving face-to-face counseling alone could reduce the time required to complete the informed consent process and additionally assessed whether there were any effects on patient's satisfaction and comprehension. We hypothesize that the use of a multimedia-facilitated informed consent (MIC) process will provide surgeons a method to more efficiently obtain informed consent without negatively affecting patient's satisfaction and comprehension. We also provide a discussion of the advantages to both physicians and patients.

\section{Participants and methods Participants}

Institutional Review Board approval (HS\# 2017-3626) was obtained from the University of California, Irvine, CA, USA, prior to subject recruitment and adheres to the tenets of the Declaration of Helsinki. The trial was registered under the International Standard Randomized Controlled Trials (ISRCTN 16303856). At a single center, candidates for standard cataract extraction with one surgeon were recruited for this study and verbal informed consent with an approved study information sheet was obtained for all active participants as approved by the Institutional Review Board. Eligibility criteria for this trial were patients undergoing first time counseling for phacoemulsification (phaco) cataract surgery for the placement of a monofocal lens. Exclusion criteria for patients in this study were as follows: 1) patients with prior cataract extraction in the opposing eye; 2) patients unable to view and/or hear videos played on a tablet device; 3 ) patients unable to complete the administered questionnaire without extra assistance; and 4) patients unable to read or speak English.

\section{Study design}

This study was conducted at a single center; participants meeting the above listed inclusion and exclusion criteria were randomly assigned in equal proportions to either the MIC group or the control group using Google's search engine (Google LLC). This was performed by searching the phrase "flip a coin" and assigning those with "heads" to the MIC group and those with "tails" to the control group. The participating surgeon in this study was masked to the randomization assignment for all participants.

The MIC group was shown a cataract surgery education video prior to receiving counseling, whereas the control group received only traditional face-to-face counseling with the surgeon. The proposed cataract surgery education video for this study, entitled "Phaco Cataract Surgery with Monofocal Lens" was obtained from the American Academy of Ophthalmology - Academy Store as one of the 21 videos included in the Cataract Collection. ${ }^{12}$ These educational videos meet the Ophthalmic Mutual Insurance Company's standards for informed consent messaging. The video used was not modified in any way and was shown for the full run time of 4 minutes and 9 seconds to each participant assigned to the MIC group.

Participants in the MIC group were placed in a quiet room for viewing the video immediately prior to meeting the surgeon. The video was administered using a LG G Pad (LG Corporation, Seoul, South Korea) tablet device set to maximum brightness and maximum volume. Participants were asked to confirm that the video content was both clearly visible and audible before proceeding with viewing. Following completion of the video, participants then met with the primary surgeon for standard counseling to complete the informed consent process. Participants in the control group received only standard face-to-face counseling to complete the informed consent process.

The length of time required by the primary surgeon to complete the consent process was measured for subjects in both the MIC and the control groups. Participants were then handed a self-administered questionnaire to evaluate satisfaction and comprehension (Figure 1).

The length of time to complete the informed consent process served as the primary outcome measure for this study, with the questionnaire serving to provide measures for assessing satisfaction and comprehension. An independent researcher was enlisted to recruit subjects that met the appropriate criteria listed, randomize the subjects to each group, assist with playing the video for subjects, record the length of time during subject counseling, and administer the questionnaire at the conclusion of each visit.

\section{Statistical analysis}

After consulting our departmental biostatistician group, the targeted sample size for this study was 60 participants (30 participants per group). A smaller sample size was chosen 


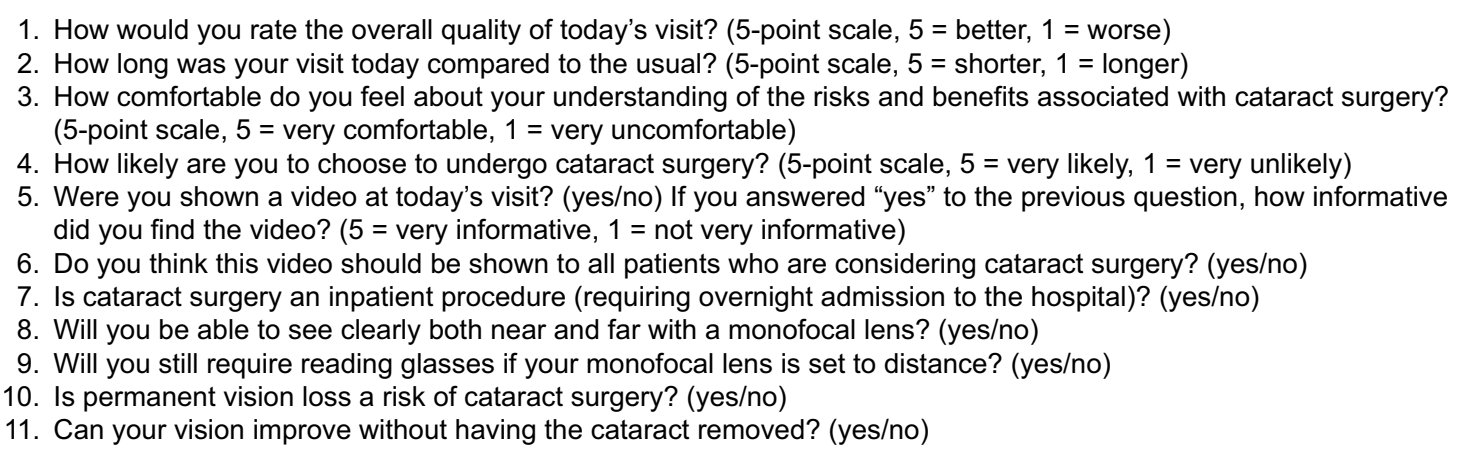

Figure I Self-administered questionnaire given to patients undergoing phacoemulsification cataract surgery for the placement of a monofocal lens.

as no follow-up was required, and therefore, no participant dropouts were expected during the study. Statistical analysis was performed using the GraphPad Prism 7.0 software (GraphPad Software, Inc., La Jolla, CA, USA). For the comprehension portion of the questionnaire, a correct answer was assigned a score of 1 and an incorrect answer received a score of 0 . Final scores were totaled and represented as a percentage of correct answers. The remaining questions, including those for measuring satisfaction, were represented on a 5-point scale (Figure 1). Statistical testing was performed with an unpaired $t$-test using the GraphPad software, the cutoff for significance set at $P<0.05$. Mean \pm standard error of mean was calculated and reported where appropriate.

\section{Results}

A total of 86 individuals were considered for this study with 63 individuals meeting the appropriate criteria for enrollment; 33 individuals were randomized to be in the MIC group and 30 individuals were randomized to serve as the control group (baseline demographics are presented in Table 1 with Figure 2 showing subject flow during the study). Compared to the control group, the MIC group demonstrated similar satisfaction $(4.67 \pm 0.104$ video versus $4.53 \pm 0.133$ control; $P=0.43)$ and comprehension $(79.4 \% \pm 2.82 \%$ video versus $79.3 \% \pm 3.39 \%$ control; $P=0.99$ ) (Tables 2 and 3 ). Both the

Table I Baseline characteristics of all enrolled participants

\begin{tabular}{lll}
\hline Characteristics & \multicolumn{2}{l}{ Group } \\
\cline { 2 - 3 } & MIC $(\mathbf{n}=\mathbf{3 3})$ & Control $(\mathbf{n}=\mathbf{3 0})$ \\
\hline $\begin{array}{l}\text { Age }(\%) \\
<70 \text { years }\end{array}$ & $8(24.2)$ & $11(36.6)$ \\
$\geq 70$ years & $25(75.6)$ & $19(63.3)$ \\
Race & & \\
$\quad$ Caucasian & $21(63.6)$ & $23(76.6)$ \\
Hispanic & $7(21.2)$ & $5(16.6)$ \\
African American & $3(9.09)$ & $2(6.67)$ \\
Asian & $2(6.06)$ & 0 \\
\hline
\end{tabular}

Abbreviation: MIC, multimedia-facilitated informed consent.
MIC group and control group also felt similarly about length of the visit $(3.33 \pm 0.207$ video versus $3.50 \pm 0.248$ control; $P=0.61)$, comfortability with the risks/benefits associated with surgery $(4.73 \pm 0.100$ video versus $4.67 \pm 0.100$ control; $P=0.67)$, and likelihood to undergo surgery $(4.82 \pm 0.081$ video versus $4.80 \pm 0.074$ control; $P=0.87$ ). Counseling time, however, was significantly lower in the MIC group (117.5 \pm 10.9 seconds video versus $241.6 \pm 13.0$ seconds control; $P<0.0001)$. Additionally, all subjects in the MIC group found the video to be "very informative" and responded "yes" when asked whether they thought that the video should be shown to all patients considering cataract surgery.

\section{Discussion}

Patient's education videos are becoming more widely used and increasingly popular because they allow important information to be explained in a succinct but easily understandable way. However, studies attempting formal evaluation of their application remain limited. During the design of this study, it was important to first establish what outcome measures were important for the patient informed consent process, which was narrowed to the length of time, patient's satisfaction, and patient's comprehension. Compromise to any of these core components, especially patient's satisfaction and comprehension, could result in more harm than any benefit gained from increased efficiency of the informed consent process. The study conducted was intended to show that the use of patient's education videos, while unlikely able to completely replace traditional face-to-face counseling, can be used effectively as a supportive tool in assisting the face-to-face informed consent process for cataract surgery.

In our study, the comprehension and satisfaction levels between the MIC and control groups were not significantly different. This is consistent with the study by Johnson et al, ${ }^{13}$ who reported no difference in comprehension or satisfaction when looking at groups receiving information about total 


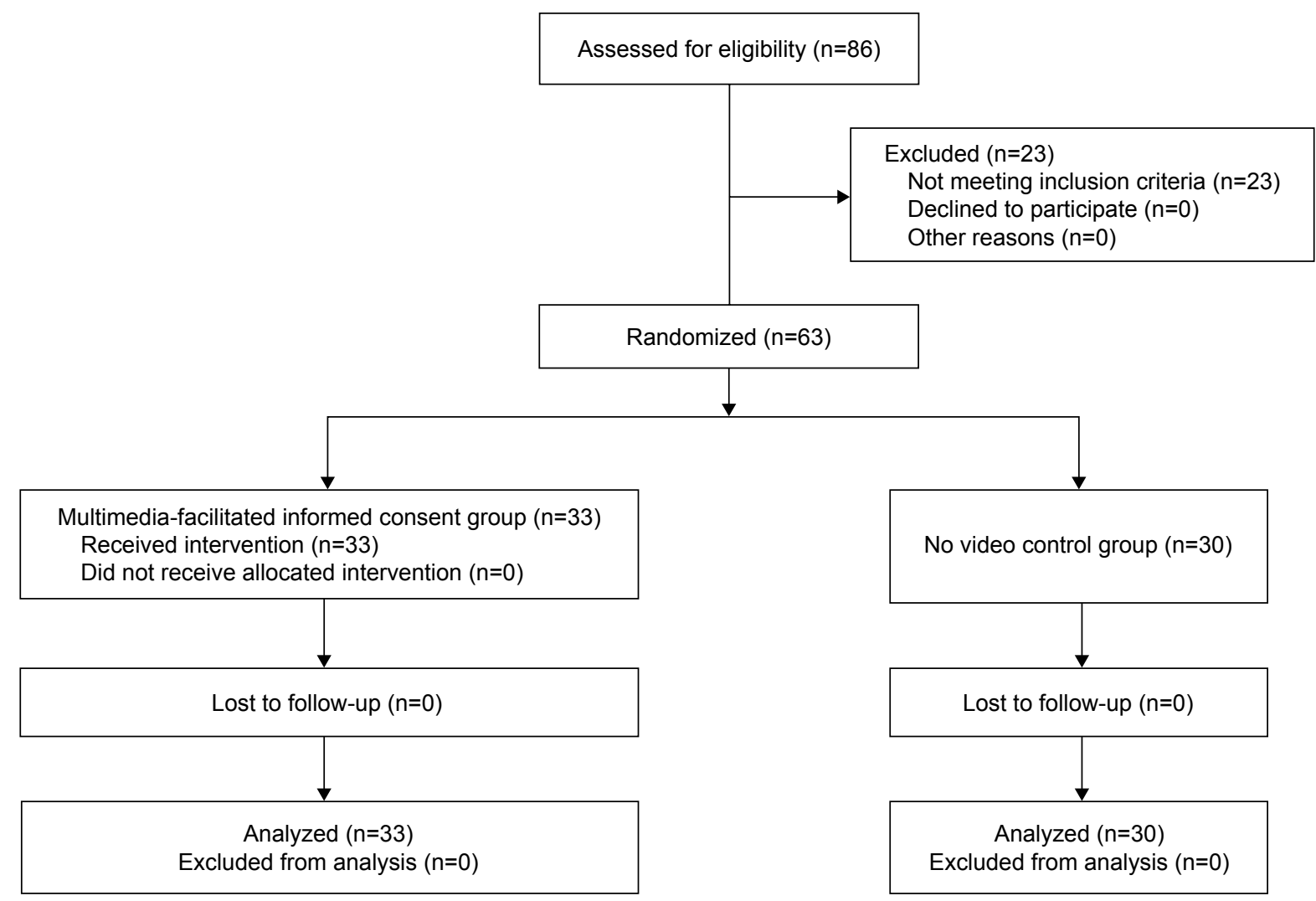

Figure 2 Flowchart of participants enrolled in the study.

Note: Given the duration of this trial, no participants were lost to follow-up.

Table 2 Overall assessment of visit quality self-reported by participants and time recorded to complete the consent process

\begin{tabular}{|c|c|c|c|}
\hline \multirow[t]{2}{*}{ Question } & \multicolumn{2}{|l|}{ Group } & \multirow[t]{2}{*}{$P$-value } \\
\hline & MIC & Control & \\
\hline How would you rate the overall quality of today's visit? ( $5=$ best, $I=$ worst $)$ & $4.67 \pm 0.104$ & $4.53 \pm 0.133$ & 0.43 \\
\hline How long was your visit today compared to the usual? ( 5 = shorter, I = longer) & $3.33 \pm 0.207$ & $3.50 \pm 0.248$ & 0.61 \\
\hline $\begin{array}{l}\text { How comfortable do you feel about your understanding of the risks and benefits associated } \\
\text { with cataract surgery? ( } 5 \text { = very comfortable, I = very uncomfortable) }\end{array}$ & $4.73 \pm 0.100$ & $4.67 \pm 0.100$ & 0.67 \\
\hline How likely are you to choose to undergo cataract surgery? $(5=$ very likely, $I=$ very unlikely $)$ & $4.82 \pm 0.081$ & $4.80 \pm 0.074$ & 0.87 \\
\hline How informative did you find the video? ( $5=$ very informative, $I=$ very uninformative) & 5 & $\mathrm{~N} / \mathrm{A}$ & \\
\hline Time to complete the consent process (seconds) & $117.5 \pm 10.9$ & $241.6 \pm 13.0$ & $<0.0001$ \\
\hline
\end{tabular}

Abbreviations: MIC, multimedia-facilitated informed consent; N/A, not applicable.

Table 3 Percentage of correct responses for each item on the comprehension portion of the questionnaire, $P=0.99$

\begin{tabular}{lll}
\hline Question (correct answer) & Group \\
\cline { 2 - 3 } & MIC (\%) & Control (\%) \\
\hline 1) Is cataract surgery an inpatient procedure (requiring overnight admission to the hospital)? (no) & 90.0 & 93.9 \\
2) Will you be able to see clearly both near and far with a monofocal lens? (no) & 53.3 & 42.4 \\
3) Will you still require reading glasses if your monofocal lens is set to distance? (yes) & 86.7 & 84.8 \\
4) Is permanent vision loss a risk of cataract surgery? (yes) & 87.9 & 80.0 \\
5) Can your vision improve without having the cataract removed? (no) & 81.8 & 83.3 \\
Overall & $79.4 \pm 2.82$ & $79.3 \pm 3.39$ \\
\hline
\end{tabular}

Abbreviation: MIC, multimedia-facilitated informed consent. 
knee arthroplasty through video. ${ }^{13}$ However, there is some disparity in the literature, as several other studies found significant improvements in comprehension for those receiving video-based information. ${ }^{8-11}$ Zhang et al, ${ }^{8}$ who performed a similar study examining cataract surgery candidates, reported a satisfaction of $86 \%$ for those who viewed a video and $65 \%$ $(P=0.035)$ for those who did not. These variable findings may be attributed to external factors such as quality of the video used, educational level of the patient population sampled, and methods for evaluating outcome measures. Satisfaction, for example, is subjective and relies on self-reported assessments and therefore cannot be reliably quantitated compared to other outcome measures. Nonetheless, no studies reported that the use of patient's education videos resulted in a decreased patient's satisfaction or comprehension..$^{8-11,13}$ This finding suggests that the incorporation of educational videos into the informed consent process can be equally as effective as traditional methods of surgical counseling. In our study, we also found that the amount of time required to complete the consent process for the MIC group was significantly lower than the control group, suggesting that videos can help improve efficiency. The subjects who viewed the video were also in full consensus that the cataract video should be shown to all patients considering surgery.

The incorporation of patient's education videos into the informed consent process provides other inherent advantages that were not specifically assessed. For example, it was observed that in the MIC group, counseling from the surgeon was directed at more focused and nuanced questions or concerns specific to each patient. Videos also offer the advantage of being paused or replayed at a patient's leisure and can be broadcasted in a waiting room during idle wait times, which may decrease patients' perception of waiting times. Providing patients access to these education videos prior to meeting with their surgeon could prove beneficial by offering patients more time to carefully formulate questions and concerns that can be addressed by their surgeon.

Arguably, the most valuable advantage that these videos offer though is that they ensure a consistent, simplified, explanation of cataract surgery and the associated risks and benefits. A well-prepared video can prevent any confusion or misunderstandings regarding surgery, which is critical not only to educate patients but also to help protect physicians, especially from a documentation and medicolegal perspective. Among ophthalmologists, one-third of all malpractice litigations involve cataract surgery. ${ }^{14}$ And in $90 \%$ of all ophthalmologic malpractice cases, inadequate or lack of informed consent is listed as a supporting cause. ${ }^{14}$ Videos can help to standardize the information presented, ensure that essential information is disseminated, and avoid any misrepresentation of cataract surgery. This may be especially valuable in an academic setting, where surgical trainees may not yet have adequate experience with appropriately guiding patients through the informed consent process.

There were several limitations observed during this study. The use of "yes/no" responses on the questionnaire lacks the degree of accuracy that free response questions would provide when assessing comprehension. While the use of yes/ no response is appropriate for certain questions, the addition of free-response questions could help improve the quality of the questionnaire. Background knowledge regarding cataract surgery was also not assessed prior to enrollment. Given the myriad of sources available online, it is not unexpected that some participants would already have a basic understanding of cataract surgery prior to their enrollment in the study, skewing the counseling time and comprehension level. Establishing a baseline knowledge level by administering a questionnaire both before and after evaluation can circumvent this issue. This study also utilized one primary surgeon for evaluating patients to ensure that both the MIC and control groups received identical counseling. A larger study, encompassing more participants and more surgeons, could strengthen the power of this study.

Future studies on this subject could also aim to evaluate the duration of knowledge retention. Videos serve as both visual and auditory media for presenting pertinent information to the patients, which may enhance patient's comprehension of the information provided. ${ }^{9}$ This could be assessed by administering a postoperative questionnaire during follow-up visits.

\section{Conclusion}

We believe that these results are promising and support the application of patient's education videos where appropriate. While not intended to replace traditional face-to-face counseling with a surgeon, carefully produced patient's education videos, such as those provided by the American Academy of Ophthalmology, offer an additional method of educating patients about cataract surgery and establishing realistic expectations for outcomes. When used as an adjunct, these videos maintain a consistent, simplified delivery of information and can help avoid misunderstandings that may otherwise occur during the informed consent process. Given the fact that $30 \%$ of all ophthalmic malpractice cases involve 
cataract surgery and $90 \%$ of malpractice cases involve inappropriate or inadequate informed consent, an improved multimedia-assisted informed consent process is important to protect not only patients but also health care providers.

\section{Acknowledgments}

The authors would like to thank Research to Prevent Blindness (RPB) for their continued support of our institution, faculty, residents, and medical students. This study was supported by the RPB Medical Student Eye Research Fellowship and the Gavin Herbert Eye Institute, which is an institutional RPB grant recipient. The funding organization had no role in the design or conduct of this research.

\section{Disclosure}

The authors report no conflicts of interest in this work.

\section{References}

1. World Health Organization [webpage on the Internet]. Priority Eye Diseases. World Health Organization; 2017. Available from: http:// www.who.int/blindness/causes/priority/en/index1.html. Accessed April $25,2017$.

2. National Institutes of Health. Facts about Cataract. U.S. Department of Health and Human Services; 2015. Available from: https://nei.nih.gov/ health/cataract/cataract_facts. Accessed April 25, 2017.

3. Ellwein LB, Urato CJ. Use of eye care and associated charges among medicare population 1991-1998. Arch Ophthalmol. 2002;120(6): 804-811.

4. Betti S, Sironi A, Saino G, Ricci C, Bonavina L. Effect of the informed consent process on anxiety and comprehension of patients undergoing esophageal and gastrointestinal surgery. J Gastrointest Surg. 2011;15(6): 922-927.
5. Migden M, Chavez-Frazier A, Nguyen T. The use of high definition video modules for delivery of informed consent and wound care education in the Mohs surgery unit. Semin Cutan Med Surg. 2008;27(1):89-93.

6. Tan JF, Tay LK, Ng LH. Video compact discs for patient education: reducing anxiety prior to cataract surgery. Insight. 2005;30(4):16-21.

7. Hoppe DJ, Denkers M, Hoppe FM, Wong IH. The use of video before arthroscopic shoulder surgery to enhance patient recall and satisfaction: a randomized-controlled study. J Should Elbow Surg. 2014; 23(6):e134-e139.

8. Zhang Y, Ruan X, Tang H, Yang W, Xian Z, Lu M. Video-assisted informed consent for cataract surgery: a randomized controlled trial. J Ophthalmol. 2017;2017:9593631.

9. Shukla AN, Daly MK, Legutko P. Informed consent for cataract surgery: patient understanding of verbal, written, and videotaped information. $J$ Cataract Refract Surg. 2012;38(1):80.

10. Moseley TH, Wiggins MN, O'Sullivan P. Effects of presentation method on the understanding of informed consent. Br J Ophthalmol. 2006;90(8):990-993.

11. Sanchez JP, Kaltwassar S, McClellan M, Burton WB, Blank A, Calderon Y. Educational video tool to increase syphilis knowledge among black and Hispanic male patients. J Health Care Poor Underserved. 2010;21(1):371-385.

12. American Academy of Ophthalmology. Phaco Cataract Surgery with Monofocal Lens. Cataract and Refractive Surgery Patient Education Video Collection. 2017.

13. Johnson MR, Singh JA, Stewart T, Gioe TJ. Patient understanding and satisfaction in informed consent for total knee arthroplasty: a randomized study. Arthritis Care Res (Hoboken). 2011;63(7):1048-1054.

14. Kiss CG, Richter-Mueksch S, Stifter E, Diendorfer-Radner G, VelikayParel M, Radner W. Informed consent and decision making by cataract patients. Arch Ophthalmol. 2004;122(1):94-98.
Clinical Ophthalmology

\section{Publish your work in this journal}

Clinical Ophthalmology is an international, peer-reviewed journal covering all subspecialties within ophthalmology. Key topics include: Optometry; Visual science; Pharmacology and drug therapy in eye diseases; Basic Sciences; Primary and Secondary eye care; Patient Safety and Quality of Care Improvements. This journal is indexed on Submit your manuscript here: http://www.dovepress.com/clinical-ophthalmology-journal

\section{Dovepress}

PubMed Central and CAS, and is the official journal of The Society of Clinical Ophthalmology (SCO). The manuscript management system is completely online and includes a very quick and fair peer-review system, which is all easy to use. Visit http://www.dovepress.com/ testimonials.php to read real quotes from published authors. 